\title{
Sociocultural context of emotional intelligence development of 5-7 year old children.
}

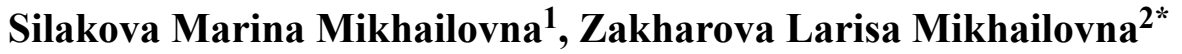 \\ ${ }^{1}$ Department of Psychology, Ulyanovsk State Pedagogical University, Ulyanovsk, Russia \\ ${ }^{2}$ Department of Preschool and Primary Education, Ulyanovsk State Pedagogical University, Ulyanovsk, Russia
}

\begin{abstract}
Sociocultural context of the development of emotional intelligence development of 5-7 year old children. Emotional intelligence is an important component in the process of a person's life; it affects the effectiveness of his relationship with the others and determines his emotional well-being. Emotional intelligence includes two main characteristics: the recognition of emotions and their expression. The expression of emotions is related to the culture within which the child is brought up. The study, which involved 5-7 year old children living in different sociocultural settings (orphanages and families), showed that, while recognizing the emotions; the children of both groups correctly determined only the basic emotions. The emotions that are the "product" of social relations (offense, pensive) were best determined by children living in families. Verbal descriptions of emotions and the prediction (regulation) of further behaviour based on emotions were also better implemented by the children raised in families. The results of the pilot study suggest that the distinction and description of emotions, as a part of emotional intelligence, by 5-7 year old children depends on the social experience obtained in a particular environment of upbringing.
\end{abstract}

Keywords: Emotional intelligence, Emotion recognition, Socio-cultural environment, Basic emotions, Emotional regulation.

Accepted date: January 12, 2018

\section{Introduction}

Emotional culture of a man is an important component in any society. Emotions are the first "identifying" signal in the establishment of the interaction among people, recognition by the "familiar-unfamiliar" type, and the construction of a further behaviour strategy.

In the modern philosophical studies, the role of emotional competence as the openness of a person to one's emotional experience is emphasized $[1,2]$, it is also considered within a harmonious connection between affect and intellect, heart and reason. One more important problem of the modern society is the problem of expressing emotions and managing them. The ban on the bright manifestation of emotions in many cultures often leads to their displacement from consciousness, the inability to respond and, as a consequence, the strengthening of the physiological component leading to neurosis. On the other hand, emotions that is out of control lead to negative consequences in the system of interpersonal relationships, experiences, which can also lead to deterioration of mental and physical health [3-5].

Emotions in various content contexts are constantly studied by researchers around the world. The particular attention is paid to the relative development of emotions and their types, the regulation of the emotional sphere, emotional intelligence, etc. The concept of emotional intelligence, developed in the works of Mayer et al. [6], Goleman [7] and Bar-On [8] makes it possible, in our opinion, to understand the manifestations and regulation of emotions from the point of view of the sociocultural environment. It is related to the knowledge of one's own emotional states and emotional states of other people, and the use of these states for successful interaction. In this regard, the most important structural components of emotional intelligence are the understanding and recognizing the emotions, their expression in accordance with cultural patterns adopted in a society and behaving according to everything mentioned above.

Undoubtedly, at the preschool age there is a specific manifestation of emotional intelligence. It is closely connected with the age specific features within the development of mental functions and the initial stages of socialization associated with the culture of the society in which the child is raised (there takes place the socialization of the emotional sphere).

\section{Conceptual frame of study}

Socialization of the emotional sphere of a child is connected with the mechanisms of emotional synchrony and emotional decentration. The mechanism of emotional synchrony usually dominates the children under the age of 3 and at the age of 5-7 the socialization of the emotional sphere follows the mechanism of emotional decentration. The essence of this mechanism is the emotional switching from oneself to the others, and it underlies the emergence of such social forms of interaction as empathy, sympathy [9]. Another aspect of emotional intelligence, connected with the regulation of emotions, has also been repeatedly discussed. LaFreniere [10], Boyd et al. [11] and Davies [12], consider regulation as an 
internal mechanism by which one can control one's emotions, as the presence of an emotional anticipation, which is based on the connection between affect and intelligence, which allows the child to analyze the reasons that caused the emotional balance or some other emotional reaction and to regulate their own emotions $[13,14]$.

There is one Russian psychological research, which deserves our particular attention. It is devoted to the creation of a level model of the affective organization of consciousness and behavior. It was proposed by Nikolskaya [15] and later developed in the works of Bardyshevskaya and Lebedinsky [16], Semago and Semago [17]. Nikolskaya [15] writes about the cultural development of the affective sphere. During the process of interaction with the environment there appear the new types of experiences, in which the affective impulses of a child begin to be contrasted to the culture or the system of social relations.

Within the period of preschool childhood there is an intensive process of developing self-awareness, reflexive skills, decentration, which allows creating conditions for the development of emotional intelligence of preschool children.

Emotional intelligence is formed in the conditions of the sociocultural specificity of the society, and is based on the values of people's livelihoods. Approved behaviour is correlated with normative standards and the image of culture in micro- and macro-levels of sociocultural environment.

The conditions of family education (micro society) daily allow a child to feel and understand how his behavior affects the emotional state of his close people: parents, sisters and brothers. Emotional content of joint actions expands the spectrum of emotional manifestations of a child. Children left without parental care are brought up in the orphanage. In our opinion, the opportunities for developing sensitivity to the needs of the others are limited.

\section{Purpose of research}

To determine whether the socio-cultural context of upbringing (family and orphanage) affects the development of the emotional intelligence of 5-7 year old children.

To distinguish whether there exist any difference in emotional states of children living with their parents or of children who are brought up in an orphanage.

\section{Describing Sample, Procedure and Methods of Investigation}

The pilot survey involved 30 5-7-year-old children attending a kindergarten, as well as 30 5-7 year old children living in an orphanage. The survey was conducted in Ulyanovsk region (Russia) in March, 2017.

Methods for the study were selected in accordance with the basic structural components of emotional intelligence. The study used the method "Emotional faces" [17], designed to assess the possibility of an adequate identification of the emotional state. The technique is used to work with children and teenagers. As a stimulus material, in the technique there were used two series of images of emotional facial expressions.

The first series (3 images) consists of contour (schematic) images expressing anger, sadness and joy. Emotional expression on them is indicated by the most ethologically significant elements of the face: the spatial arrangement of the corners of the lips and the position of the eyebrows.

The second series (14 images) includes images of specific faces of children (boys and girls, 7 images each). The technique uses photographs with the following emotional expressions: sheer joy, fear, anger, affability, surprise, resentfulness, pensive.

\section{Discussing the Results}

The first series of studies related to recognizing people's emotions according to schematic images showed that most children from both groups correctly named a picture showing the emotion of "joy" and the image of "anger". Average figures considering the picture "joy" are 2.5 (children from the orphanages) and 2.7 (children from the families), in both groups the average figures considering the picture "anger" is 2.3 .

The second series of studies, related to the recognition of children's emotions in pictures, showed that children from the orphanage have the highest average indices for recognizing and verbal expressing of "sheer joy" (indicator 2.4) and "fear" (indicator 2), and children from the families recognize "offense" (2.4), "affability" (2.2), "sheer joy" (indicator 2.1) and "fear" (indicator 2) with the following indices (Table 1).

Table 1. Average recognition of emotions by preschool children, who are brought up in an orphanage or in a family, in 2 series.

\begin{tabular}{|c|c|c|c|c|c|c|c|c|c|c|c|c|}
\hline \multirow{2}{*}{ Sample } & & \multicolumn{3}{|c|}{ 1st series } & \multicolumn{8}{|l|}{ 2nd series } \\
\hline & & Anger & Sorrow & Joy & Sheer joy & Fear & Gruffness & Affability & Surprise & Resentment & Pensive & Average \\
\hline $\begin{array}{l}\text { Children } \\
\text { orphanages }\end{array}$ & from & 2.3 & 1.7 & 2.5 & 2.4 & 2 & 1.7 & 2 & 1.9 & 1.9 & 1.7 & 2 \\
\hline $\begin{array}{l}\text { Children } \\
\text { families }\end{array}$ & from & 2.3 & 1.8 & 2.7 & 2.1 & 2 & 2.1 & 2.2 & 1.9 & 2.4 & 2.1 & 2.2 \\
\hline
\end{tabular}

When comparing the average indicators of recognizing emotions and their verbal designing, the particular attention is drawn to the fact that children from the orphanage have higher indices of "joy", "anger", "gruffness" and the scatter of the indicators themselves is more pronounced and the children from families have higher indices of "joy" and "resentment", 
Citation: Silakova MM, Zakharova LM. Sociocultural context of emotional intelligence development of 5-7 year old children. J Psychol

the range of indicators is less pronounced, when compared with the first group.

The "resentment" of children who are brought up at home can be viewed as a social emotion that arose in response to the lack of understanding and empathy provided by adults. Both understanding and empathy are related to close family relationships.

The analysis of children's answers to the question why they think the pictures show one or another emotion depicts the connection of vivid mimic manifestations with the corresponding emotion in the children's responses. So, joy was recognized because of a smile, "anger" and "gruffness" was recognised because of the bright, characteristic expression of the eyes and the mouth. The rest of the emotions presented in the pictures do not have such vivid distinctive features that would "connect" them to a certain emotion, so the children in both groups recognized them using additional questions, the essence of the questions was to show the relationship between the depicted emotion and typical situations in which the child could have experienced this emotion.

To understand the degree of awareness of calling the emotions by children, there was conducted an additional conversation with all the children. There were given the tasks suggesting that children should depict the emotions presented in the pictures. The observations showed that $75 \%$ of children brought up in families easily coped with tasks, concentrating their efforts on changing the line of eyebrows, corners of the lips. To strengthen the emotional expression, children used gestures such as folded hands (anger); intonational and mimic means (voice). While looking at the pictures $60 \%$ of children brought up in families and attending a kindergarten, describe possible situations that provoked these emotions and predicted possible actions to change them. "The girl is crying, it's likely that someone scolded her, offended her"; "You need to buy the same toys for everyone" "You need to share and play in turns," etc. The children who were brought up in the orphanages were able to demonstrate the basic emotions and predicted actions mainly related to the basic emotions.

The comparative analysis of the emotions recognition within two groups of children using the Mann-Whitney U-test criterion $(p<0.05)$ showed that children from the orphanages and children brought up in families equally recognize the basic emotions of the mimic facial expression (joy, fear), as well as the emotions that are close in the mimic expression of the face to the basic ones (anger, surprise, sadness), because they have similar expressions of the eyes, eyebrows and lips, typical for many expressions of emotion (for example, large eyes can be a sign of fear, anger and surprise at the same time).

The obtained data of the pilot study correlate with the results obtained by Matsumoto [18] and Ekman [19] who received data on the universality of the main emotions (Table 2).

Table 2. Reliable differences in the criterion of the Mann-Whitney U-test $(p<0.05)$ were obtained only regarding the recognition of emotions "offense" and "pensive".

\begin{tabular}{|c|c|c|c|}
\hline Recognized emotions & U-test empirical & U-test for significance $p<0.05$ & Contact with the significance of the area \\
\hline Anger & 112.5 & 72 & No \\
\hline Sorrow & 107.5 & 72 & No \\
\hline Joy & 97.5 & 72 & No \\
\hline Sheer joy & 85.5 & 72 & No \\
\hline Fear & 105.5 & 72 & No \\
\hline Gruffness & 75.5 & 72 & No \\
\hline Affability & 97.5 & 72 & No \\
\hline Surprise & 107.5 & 72 & No \\
\hline Offense & 58.5 & 72 & Yes \\
\hline Pensive & 70 & 72 & Yes \\
\hline $\begin{array}{l}\text { General level of emotion } \\
\text { recognition }\end{array}$ & 52.5 & 72 & Yes \\
\hline
\end{tabular}

These two social emotions are understood and manifested differently if children live in different conditions. Resentment arises as a result of an action/lack of some action, a word, etc. But one and the same action or word may not only cause feelings of resentment, sometimes children do not feel it because they do not have rigid expectations about the behavior of the others. It may be considered as a characteristic of an orphanage.
Offense is the emotion born within the system of significant social relations, which, first of all, are created in the family. A child who is brought up in the family, experiencing love and joy because of this love, in case of dissatisfaction, may feel insulted.

Pensive is not a basic emotion. It is characterized by the expression of negation or judgment. It is not characterized by vivid mimic manifestations. This emotion is difficult enough 
for children to recognize. At the same time, the repeated correlation of the family member's condition with his verbal designation (pensive) allows children to learn not only to recognize this emotion, but also to regulate their behavior in accordance to a specific situation. In the conditions of the orphanage, such opportunities are rather limited.

Apparently, we can assume that the distinction and management of social emotions of 5-7 year old children is based on the mechanism of empathy. It is associated with the peculiarities of the micro and macro levels of sociocultural environment.

\section{Conclusion}

Thus, according to the study the following conclusion can be drawn:

In the preschool age different components of emotional intelligence are developed to varying degrees. It is better to develop the ability to determine the emotional state by external signs, to understand verbal expression in the context of a specific situation; or which is worse to develop the ability to understand the logic of developing the situation of interpersonal interaction;

The socialization of the emotional sphere occurs as the child acquires the norms and culture of the society; understands the role of one's own emotional state and the emotions of another person while implementing communication;

Socio-cultural context of emotional intelligence is revealed in the realization of children's skills to analyze the causes that contribute to the emergence of specific emotions, the ability to regulate their own emotions within the cultural dialogue;

Features of recognizing the emotions by children brought up in different sociocultural conditions (orphanages and families) show the same recognition level of basic emotions and better recognition of some peculiar emotions (especially offense, pensive) by children who are brought up in the families.

Verbal descriptions of emotions and the prediction (regulation) of further behavior based on emotions were also better implemented by the children raised in families.

The nature of communication in the family determines the specificity of the expressive behavior of a child and his mimic manifestations. The limited and specific nature of social contacts (in orphanages) do not allow children to live in the same range of social emotions that are associated with the closeness of children and parents and, accordingly, demonstrate close emotional relations. In this regard, the sociocultural context of the upbringing of children in a kindergarten and family environment can be called a restrained factor in the emotional manifestations of children and the development of emotional intelligence.

The results of the pilot study allow us to assume the influence of the sociocultural context on emotional intelligence according to the features of recognition and manifestation of emotions that are shown by 5-7 year old children, still this assumption requires the further study.

\section{References}

1. Slepkova VI, de Vries-Geervilet L. Development of emotional competence by means of psychological training: Actual problems of crisis psychology. Minsk. 1997;130-7.

2. Kunitsyna VN, Kazarinova NV, Pogolsha VM. Interpersonal communication. St. Petersburg, Publishing house, Piter. 2001

3. Weissbach H, Dax W. Emotional intelligence. Lik Press, Moscow. 1998.

4. Orme G. Emotional thinking as a tool for achieving success. PCB, Moscow. 2003.

5. Kholmogorova AB, Garanyan NG. Culture, emotions and mental health: Psychological questions. 1999;2:61-74.

6. Mayer JD, Caruso DR, SaloveP. Emotional Intelligence meets traditional standards for an intelligence. Intelligence. 1999;27:267-98.

7. Goleman D. Emotional intelligence. Bantam, New York. 1998.

8. Bar-On R. The bar-on emotional quotient inventory (EQi): Technical manual. Multi-health systems, Toronto. 1997.

9. Lebedinsky VV, Nikolskaya OG, Baenskaya ER, et al. Emotional disorders in childhood and their correction. Moscow, 1990.

10. LaFreniere P. Emotional development: A biosocial perspective. Saint Petersburg. 2004.

11. Boyd J, Barnett WS, Bodrova E, et al. Promoting children's social and emotional development through preschool education. 2006.

12. Davies L. The essential skill of self-control. 2001.

13. Zaporozhets AV. Selected psychological works: Mental development of the child. Pedagogika, Moscow. 1986;320.

14. Belkina VN. The problem of emotional regulation of the child's social behaviour: Preschool and younger school children problems of upbringing and education materials of the conference "Ushinsky Readings", Pedagogical Pedartment, YaSPU. Yaroslavl. 2003;116-22.

15. Nikolskaya OS. Affective sphere of a person: A look through the prism of children's autism. Center of Curative Pedagogy, Moscow. 2000.

16. Bardyshevskaya MK, Lebedinsky VV. Diagnosis of emotional disorders in children. Psychology, Moscow. 2003.

17. Semago NY, Semago MM. Problem children: Basics of diagnostic and correctional work of a psychologist. Publishing house ARCTI, Moscow. 2000.

18. Matsumoto D. Culture and psychology. Publishing house Prime-Evroznak. 2002;416.

19. Ekman P. Emotions revealed: Recognizing faces and feelings to improve communication and emotional life. Publishing house Piter. 2017;336.

\section{*Correspondence to:}

Zakharova Larisa Mikhailovna,

Doctor of Pedagogical Sciences, 
Citation: Silakova MM, Zakharova LM. Sociocultural context of emotional intelligence development of 5-7 year old children. J Psychol Cognition 2018;3(1):6-10.

Head of Preschool and Primary Education Department,

Ulyanovsk State Pedagogical University,

Ulyanovsk, Russia,
Tel: +7(9510) 963390;

E-mail: ulseagull@mail.ru 